\title{
Neuroimmune interactions and COVID-19 in lung transplant recipients
}

\section{Joan Arenas-Prat $\mathbb{D}$}

Neurogenic inflammation has a wellrecognized role in the regulation of the inflammatory response to a variety of noxious stimuli, including infections. At the lung level, this response is driven by the vagus nerve by means of modulation of parasympathetic pathways that can induce bronchospasm, mucus secretion, fluid extravasation and vasodilation. Besides parasympathetic vagal fibres, these noxious stimuli activate afferent sensory C-fibres and initiate defensive reflexes that ensure airway integrity; however, they can also release inflammatory neuropeptides such as substance $\mathrm{P}$ or calcitonin gene-related peptide (CGRP). In a recent Perspective (De Virgiliis, F. \& Di Giovanni, S. Lung innervation in the eye of a cytokine storm: neuroimmune interactions and COVID-19. Nat. Rev. Neurol. 16, 645-652 (2020)) $)^{1}$, Francesco De Virgiliis and Simone Di Giovanni suggest a new strategy based on targeted modulation of vagal fibres to treat the life-threatening inflammatory reaction secondary to SARS-CoV-2 infection.

According to this novel therapeutic approach, based on the concept of the neuroimmune unit, patients who have undergone lung transplantation would be expected to have more adverse outcomes from COVID-19 owing to abolition of the anti-inflammatory effect of the vagus nerve by complete denervation of the lungs ${ }^{2}$ - including sensory, sympathetic and parasympathetic fibres - as a result of the transplant surgery. Consistent with the authors' model, a recent multicentre study showed that, although lung transplant recipients have a clinical presentation of SARS-CoV-2 infection similar to that of the general population, they have a worse prognosis and higher mortality compared with non-transplanted patients ${ }^{3}$. However, lung transplantation also results in elimination of sensory C-fibres, which would block the release of inflammatory neuropeptides such as substance $P$ and CGRP. This potential anti-inflammatory effect of lung denervation might contradict the authors' model, unless there is crosstalk between vagal parasympathetic and sensory neuroimmune modulation.

Neurogenic inflammation is a key process in many inflammatory conditions and its modulation or blockade offers several treatment options. In this respect, surgical joint denervation is a well-recognized technique that improves osteoarthritic pain and synovial inflammation in the hand, where joints are densely innervated ${ }^{4,5}$. Nevertheless, in the more complex scenario of a denervated lung, the hypothetical equilibrium between vagal parasympathetic and sensory neuroimmune modulation should be taken into account, and further research is needed to comprehend the precise physiopathology and the role of both types of fibre in neurogenic lung inflammation. Vagal, sympathetic and sensory neurectomy following lung denervation could exacerbate COVID-19-associated inflammation through neuroimmune modulation but in theory could also exert anti-inflammatory effects secondary to disruption of inflammatory neuropeptide release ${ }^{6}$.
In summary, if denervation exerts antiinflammatory effects in the joints, could it have similar effects in the lungs?

There is a reply to this letter by De Virgiliis, $\mathrm{F}$. \& Di Giovanni, S. Nat. Rev. Neurol. https:// doi.org/10.1038/s41582-021-00485-w (2021).

Joan Arenas-Prat ID Pulvertaft Hand Centre, Royal Derby Hospital, Derby, UK e-mail: arenasprat@hotmail.com https://doi.org/10.1038/s41582-021-00484-x

1. De Virgiliis, F. \& Di Giovanni, S. Lung innervation in the eye of a cytokine storm: neuroimmune interactions and COVID-19. Nat. Rev. Neurol. 16, 645-652 (2020).

2. Zantah, M., Pandya, A., Marchetti, N., Jacobs, M. R. $\&$ Criner, G. J. Lung denervation and its effect on bronchial responsiveness following lung transplant surgery. Am. J. Respir. Crit. Care Med. 201, A2838 (2020).

3. Saez-Giménez, B. et al. COVID-19 in lung transplant recipients: a multicenter study. Am. J. Transplant https://doi.org/10.1111/ajt. 16364 (2020).

4. Madsen, R. J., Stone, L. A. Knapp, J. B. \& Solomon, J. S. Joint denervation in the digits: technique and patient satisfaction. Ann. Plast. Surg 80, 27-31 (2018)

5. Kane, D. et al. Protective effect of sensory denervation in inflammatory arthritis (evidence of regulatory neuroimmune pathways in the arthritic joint). Ann. Rheum. Dis. 64, 325-327 (2005).

6. O'Connor, T. M. et al. The role of substance $P$ in inflammatory disease. J. Cell. Physiol. 201, 167-180 (2004).

Competing interests

The author declares no competing interests.

\section{Reply to: Neuroimmune interactions and COVID-19 in lung transplant recipients}

\section{Francesco De Virgiliis and Simone Di Giovanni(B)}

We would like to thank Joan Arenas-Prat for his correspondence on our Perspective (Lung innervation in the eye of a cytokine storm: neuroimmune interactions and COVID-19. Nat. Rev. Neurol. 16, 645-652 (2020)) ${ }^{1}$, which raises some interesting issues (Neuroimmune interactions and COVID-19 in lung transplant recipients. Nat. Rev. Neurol. https://doi.org/ 10.1038/s41582-021-00484-x (2021)) $)^{2}$.

In our Perspective, we proposed a novel therapeutic approach to COVID-19 based on a model of neuroimmune interaction in the lungs, in which we suggest that lung inflammation is modulated by lung-innervating nerve fibres. This model implies that targeted modulation of lung innervation, especially vagal fibres, which account for the majority of lung innervation, could help resolve lifethreatening lung inflammatory reactions in pathological situations such as COVID-19.
As lung transplant surgery results in acute complete denervation of the lungs, such a condition could be of help to better characterize the clinical relevance of our neuroimmune communication model.

In his Correspondence 2 , Arenas-Prat discusses our Perspective in light of a recent publication from Saez-Giménez B. et al. ${ }^{3}$, stating that "a recent multicentre study showed that, although lung transplant recipients have a clinical presentation of SARS-CoV-2 infection similar to that of the general population, they have a worse prognosis and higher mortality compared with non-transplanted patients." However, he argues that lung transplantation will also result in the elimination of sensory C-fibres, which would block the release of inflammatory neuropeptides such as substance $\mathrm{P}$ and calcitonin gene-related peptide, and lead to the elimination of parasympathetic 
innervation, which might result in an anti-inflammatory outcome. In addition, he mentions studies in patients who underwent surgical joint denervation, which showed that loss of sensory inputs at the joint relieved osteoarthritic pain and synovial inflammation ${ }^{4,5}$. On the basis of these data, the author suggests that sensory denervation could have similar effects in the lungs, which might be at odds with our model whereby vagus innervation is proposed to have a net anti-inflammatory function.

Although we agree with the author that crosstalk between vagal parasympathetic and sensory neuroimmune modulation will be taking place, the net pro-inflammatory or anti-inflammatory outcome is likely to be tissue-specific and will depend on the inflammatory stimulus. The data from Saez-Giménez B. et al. ${ }^{3}$ provide exciting clinical evidence in support of our model, as acute lung denervation due to transplant surgery was associated with adverse outcomes from COVID-19. Indeed, in line with our proposed model, these data suggest that lack of lung innervation could worsen SARS-CoV-2 infection outcomes, mirroring the poor COVID-19 prognosis observed in elderly individuals ${ }^{6}$, in whom the vagal tone is reduced.

Although these data do not systematically prove our model, they strengthen the idea that the activity of vagal fibres innervating the lungs could be manipulated to prevent maladaptive inflammation in patients affected by COVID-19, and possibly by other respiratory viruses.

Lastly and most importantly, these findings strongly advocate the urgent need for systematic studies to shed light on fibre-specific tissue innervation and its interaction with the immune system in tissue-specific pathophysiological inflammatory conditions, including but not limited to COVID-19.
Francesco De Virgiliis and Simone Di Giovanni (D) Department of Brain Sciences, Division of Neuroscience, Imperial College London, London, United Kingdom. 凶e-mail: s.di-giovanni@imperial.ac.uk

https://doi.org/10.1038/s41582-021-00485-w

1. De Virgiliis, F. \& Di Giovanni, S. Lung innervation in the eye of a cytokine storm: neuroimmune interactions and COVID-19. Nat. Rev. Neurol. 16, 645-652 (2020).

2. Arenas-Prat, J. Neuroimmune interactions and COVID-19 in lung transplant recipients. Nat. Rev. Neurol. https:// doi.org/10.1038/s41582-021-00484-x (2021).

3. Saez-Giménez, B. et al. COVID-19 in lung transplant recipients: a multicenter study. Am. J. Transplant. https://doi.org/10.1111/ajt.16364 (2020).

4. Kane, D. et al. Protective effect of sensory denervation in inflammatory arthritis (evidence of regulatory neuroimmune pathways in the arthritic joint). Ann. Rheum. Dis. 64, 325-327 (2005).

5. Madsen, R. J., Stone, L. A., Knapp, J. B. \& Solomon, J. S Joint denervation in the digits: technique and patient satisfaction. Ann. Plast. Surg. 80, 27-31 (2018).

6. Navaratnam, A. V., Gray, W. K., Day, J., Wendon, J. $\&$ Briggs, T. W. R. Patient factors and temporal trends associated with COVID-19 in-hospital mortality in England: an observational study using administrative data. Lancet Respir. Med. https://doi.org/10.1016/ S2213-2600(20)30579-8 (2021).

Competing interests

The authors declare no competing interests. 